\title{
Clinical and Pathological Findings of Renal Biopsy in Children: Outcomes from a Single Center Over 27 Years
}

\author{
Shin Ae Lee, M.D. ${ }^{1}$ \\ Min Sun Kim, M.D. ${ }^{1,2}$ \\ Soon Chul Kim, M.D., ${ }^{1,2}$ \\ Dae-Yeol Lee, M.D. ${ }^{1,2}$ \\ Department of Pediatrics', Chonbuk \\ National University Medical School, \\ Research Institute of Clinical Medicine \\ of Chonbuk National University- \\ Biomedical Institute ${ }^{2}$ of Chonbuk \\ National University Hospital, Jeonju, \\ Korea \\ Corresponding author: Dae-Yeol Lee, M.D. \\ Department of Pediatrics, Chonbuk Na- \\ tional University Hospital 20 Geonji-ro, \\ Deokjin-gu, Jeonju 54907, Korea \\ Tel: +82-63-250-1469 \\ Fax: +82-63-250-1464 \\ E-mail: leedy@jbnu.ac.kr
}

Received: 1 January 2017

Revised: 8 March 2017

Accepted: 10 March 2017
Purpose: To classify the results of renal biopsy in pediatric patients and to compare pathological findings with clinical features.

Methods: This study included data of 318 children who underwent renal biopsy at our hospital between December 1987 and November 2014. Biopsy specimens were examined histopathologically using light, immunofluorescence, and electron microscopy.

Results: Asymptomatic urinary abnormalities was the most common clinical diagnosis (35.9\%), followed by nephrotic syndrome (29.3\%), and acute glomerulonephritis (18.0\%). Glomerular disease was identified in $98.1 \%$ of the renal biopsy specimens. The most common primary cause of glomerulonephritis was IgA nephropathy, with gross hematuria in $61.9 \%$ of the patients, hypertension in $14.2 \%$, proteinuria $>1.0 \mathrm{gm} / 24-\mathrm{hr}$ in $33.3 \%$, and impaired renal function in $3.6 \%$ patients. Conclusion: The most common clinical diagnosis was asymptomatic urinary abnormalities, with primary glomerular disease being the most common renal biopsy finding, and IgA nephropathy the most common histopathological lesion. This study provides a 27-year overview of pediatric renal disease at our center and underlines the importance of renal biopsy for accurate diagnosis and proper management.

Key words: Clinical diagnosis, Children, Renal biopsy, Nephrotic syndrome, IgA nephropathy

\section{Introduction}

The diagnosis and treatment of pediatric kidney diseases is based on medical history and physical findings. However, in certain cases, a discrepancy between clinical and pathological findings exists, with patients exhibiting similar clinical symptoms despite different underlying pathological features. Although percutaneous renal biopsy is a widely used medical procedure for the diagnosis of kidney disease in adults, the procedure has not been considered suitable for pediatric patients. However, with recent innovations in technique for kidney localization and biopsy, percutaneous renal biopsy is now regarded to be both safe and advisable for the diagnosis of renal disease in pediatric patients and to inform treatment ${ }^{1-4)}$. The indications for renal biopsy vary according to the ethnic and age characteristics of the population studied as well as the geographic location ${ }^{5}$. 
The aim of our study was to classify results of renal biopsy in pediatric patients, based on pathological findings, and to compare the pathological diagnosis to clinical findings.

\section{Material and methods}

\section{Subjects}

A retrospective study of 345 inpatients who underwent renal biopsy in the Pediatric Department of Chonbuk National University Children's Hospital, between December 1987 and November 2014. The study adhered to the principles of the Declaration of Helsinki and was approved by the Life Science Research Ethic Council Committee of the Chonbuk National University Hospital (CUH 2016-10-009).

Among the 345 patients who underwent biopsy over the study period, 23 were excluded due to incomplete demographic or clinical data, and four due to inappropriate tissue samples ( $<5$ glomeruli in the kidney tissue sample). Prior to renal biopsy, prothrombin and bleeding/clotting time were assessed to determine bleeding tendency. Consent for the procedure was obtained from parents and/or guardians prior to the procedure.

Over the 27-year period of the study, there was considerable variation in the biopsy technique used. Early in the study period, biopsies were performed under sedation using a biopsy gun under fluoroscopy. Since 1997, biopsies were performed under sedation, using an automated gun under real time ultrasound guidance. All biopsies were performed by a pediatric nephrologist. For all biopsies, two cores of kidney tissues were obtained for histopathology assessment, by light microscopy, immunofluorescence microscopy, and electron microscopy (LM, IF, and EM, respectively).

\section{Data retrieval}

The following clinical and laboratory data were extracted from the clinical records for analysis: patient's age and sex; serum total protein, albumin, cholesterol, blood urea nitrogen (BUN), creatinine (Cr), and electrolytes; complement 3 (C3) and 4 (C4) and anti-streptolysin-O (ASO) titer; glomerular filtration rate $\left(\mathrm{mL} / \mathrm{min} / 1.73 \mathrm{~m}^{2}=\mathrm{kL} / \mathrm{Pcr}, \mathrm{k}\right.$ : $0-1$ years of age $0.45,2-12$ years of age 0.55 , Jaffe method); and $24-\mathrm{hr}$ urine protein $\left(\mathrm{mg} / \mathrm{m}^{2} / \mathrm{hr}\right)$.

\section{Pathological study}

For LM examination, 10 serial sections were cut and prepared using standard procedures and stained with hematoxylin-eosin (HE), Masson's trichrome, periodic acid-Schiff stain, and silver stains. Tissue specimens for IF examination are snap-frozen in liquid nitrogen and cut on a cryotome. The tissue was stained by the direct method using fluorescein isothiocyanate-conjugated antisera mono specific for immunoglobulin (Ig) G (IgG), IgA, IgM, C3, and fibrinogen. Tissue samples for EM examination were stained on copper 300 mesh grids with uranyl acetate and lead citrate for assessment.

\section{Statistical analyses}

Data was analyzed using SPSS software (version 22.0; IBM Corporation, Armonk, NY, USA). Simple descriptive statistics, mean and standard deviation (SD), were calculated for continuous variables, namely age, and clinical and laboratory measures. Percentages were calculated for categorical data.

\section{Results}

\section{Sex and age distribution}

Among the 318 cases included in this study, 205 were male $(64.5 \%)$ and 113 cases female (35.5\%), for a male-tofemale sex ratio of 1.8:1. The mean \pm SD age was $9.7 \pm 3.6$ years (range, 5 months to 18 years), with the following age distribution: 131 cases (41.2\%) between 11-15 years of age; 129 (40.5\%) between 6-10 years of age; 46 (14\%) between 0-5 years of age; and 12 (3.7\%) between 16-18 years of age.

\section{Indications for renal biopsy}

The indications for biopsy were as follows (Fig. 1): Recurrent macroscopic hematuria in 114 cases (35.8\%); nephrotic syndrome, with hematuria and/or hypertension at the first diagnosis, in 34 cases (10.7\%); persistent microscopic hematuria with proteinuria in 32 cases (10.1\%); and steroid resistance, steroid dependence, or frequent relapse forms of nephrotic syndrome in 32 cases (10.1\%). Persistent microscopic hematuria was detected in 23 cases (7.2\%), with positive family history, hypertension, increased urine RBC count, and parental anxiety. 


\section{Clinical diagnosis of patients before renal biopsy}

Prior to renal biopsy, the 318 cases were subdivided according to the following pre-biopsy clinical diagnoses (Table 1): 114 patients (35.9\%) with asymptomatic urinary abnormalities, among whom 44 (13.9\%) had isolated hematuria, $70(22.0 \%)$ had hematuria with proteinuria; 93 patients (29.3\%) with nephrotic syndrome; 57 patients (18.0\%) with acute glomerulonephritis; 38 patients (11.9\%) with Henoch-Schönlein purpura nephritis; 4 patients (1.2\%) with lupus nephritis; and 12 patients (3.7\%) with other conditions, such as acute renal failure, Alport syndrome, hemolytic uremic syndrome, and Korean hemorrhagic fever.

\section{Histopathological diagnosis after renal biopsy}

The distributions of histopathological diagnoses are summarized in Table 2. Glomerular disease was identified in $98.1 \%(312 / 318)$ of cases, with a diagnosis of primary glomerular disease in $85.2 \%$ (266/312) of these cases and of secondary glomerular disease in the remaining $14.7 \%$ (46/

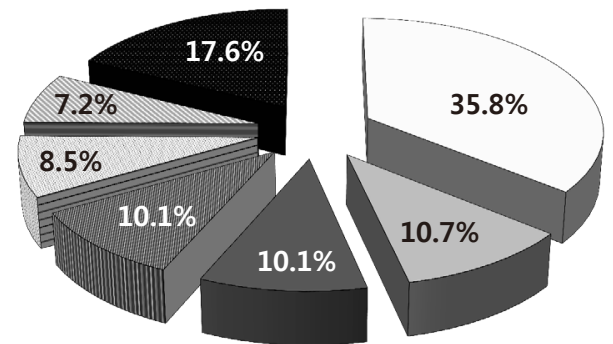

$\square$ Recurrent macroscopic hematuria

$\square$ Nephrotic syndrome associated with hematuria or hypertension $\square$ Persistent microscopic hematuria with proteinuria

m Steroid resistant, steroid dependent or frequent relapsed nephrotic syndome Evaluation of renal involvement in systemic disease

$\checkmark$ Persistent microscopic hematuria

Others

Fig1. Main indications for renal biopsy.

Table 1. Clinical Diagnosis before Renal Biopsy

\begin{tabular}{lc}
\hline Clinical diagnosis & No. of cases (\%) \\
\hline Asymptomatic urinary abnormalities & $114(35.9)$ \\
Isolated hematuria & $44(13.9)$ \\
Hematuria with proteinuria & $70(22.0)$ \\
Nephrotic syndrome & $93(29.3)$ \\
Acute glomerulonephritis & $57(18.0)$ \\
Henoch-Schönlein purpura nephritis & $38(11.9)$ \\
Lupus nephritis & $4(1.2)$ \\
Other & $12(3.7)$ \\
Total & $318(100.0)$ \\
\hline
\end{tabular}

312). Common causes of primary glomerulonephritis (GN) were IgA nephropathy, minimal change disease (MCD), and focal proliferative GN, with Henoch- Schönlein purpura nephritis being the most common cause of secondary GN.

\section{Histological classification of nephrotic syndrome}

The distribution of histological diagnoses with nephrotic syndrome is summarized in Table 3. Among the 102 cases diagnosed with nephrotic syndrome, 97 cases were histologically classified as a primary nephrotic syndrome (95.1 $\%)$ and 5 cases (4.9\%) as a secondary nephrotic syndrome. Among cases classified as a primary nephrotic syndrome, 68 cases (70.1\%) presented with MCD, with IgM nephropathy accounting for 11 of these cases (16.2\%). Focal segmental glomerulosclerosis (FSGS) was identified in 9 cases (9.3\%), membranoproliferative GN (MPGN) in 7 cases (7.2 $\%)$, IgA nephropathy in 5 cases (5.2\%), diffuse mesangial proliferative GN (DMPGN) in 4 cases (4.1\%) and membranous glomerulopathy in 4 cases (4.1\%).

Table 2. Histopathological Diagnosis after Renal Biopsy

\begin{tabular}{|c|c|c|}
\hline & Histopathology & No. of cases (\%) \\
\hline \multirow{12}{*}{$\begin{array}{l}\text { Primary } \\
\text { glomerular } \\
\text { disease } \\
(n=266)\end{array}$} & IgA nephropathy & $89(27.9)$ \\
\hline & Minimal change disease & $68(21.3)$ \\
\hline & -lgM nephropathy & 11 out of 68 \\
\hline & Focal proliferative GN & $26(8.1)$ \\
\hline & Mesangioproliferative GN & $23(7.2)$ \\
\hline & Thin GBM disease & $19(5.9)$ \\
\hline & Focal-segmental glomerulosclerosis & $11(3.4)$ \\
\hline & Postinfectious GN & $8(2.5)$ \\
\hline & Diffuse proliferative GN & $7(2.2)$ \\
\hline & Minor-Glomerular abnormalities & $7(2.2)$ \\
\hline & Alport syndrome & $4(1.2)$ \\
\hline & Membranous GN & $4(1.2)$ \\
\hline \multirow{3}{*}{$\begin{array}{l}\text { Secondary } \\
\text { glomerular } \\
\text { disease } \\
(\mathrm{n}=46)\end{array}$} & Henoch-Schönlein Purpura nephritis & $39(12.2)$ \\
\hline & Lupus nephritis & $5(1.5)$ \\
\hline & $\begin{array}{l}\text { Hepatitis B virus associated } \\
\text { membranous GN }\end{array}$ & $2(0.6)$ \\
\hline \multirow{4}{*}{$\begin{array}{l}\text { Miscellaneous } \\
(n=6)\end{array}$} & Tubulointerstitial nephritis & $3(1.0)$ \\
\hline & Acute tubular necrosis & $1(0.3)$ \\
\hline & Normal histology & $2(0.6)$ \\
\hline & Total & $318(100.0)$ \\
\hline
\end{tabular}

Abbreviations: GN, Glomerulonephritis; Thin GBM disease, Thin basement membrane disease. 


\section{Clinical status of nephrotic syndrome as glomerular morphology}

Among patients diagnosed with nephrotic syndrome, microscopic hematuria was identified in 48 patients (47.1 $\%$ ), as well as in 21 of the 68 patients (30.8\%) with MCD, 6 of the 7 patients (85.7\%) with MPGN and 6 of the 9 patients (66.7\%) with FSGS (Table 3). Microscopic hematuria was identified in all patients with membranous glomerulopathy, postinfectious glomerulonephropathy, Henoch- Schönlein purpura nephritis, and nephropathy associated with hepatitis B viral infection. Hypertension was identified in 16 patients (15.7\%), with decreased levels of serum complement in 12 patients (11.8\%), and decreased serum albumin concentration of $<2.5 \mathrm{gm} / \mathrm{dL}$ in 61 patients (59.8\%), 51 of whom (83.6\%) in the MCD group.

\section{Clinical features according to histological grades in IgA nephropathy}

Based on histological findings by Haas $\mathrm{M}^{6}$, IgA nephropathy was classified as grade I-V as in Table 4. In our study, 89 patients were diagnosed as having an IgA nephropathy following renal biopsy; a definite grade could not be established in 5 cases. The distribution of grades was as follows: 46 cases classified as grade III, 20 cases as grade IV, and 9 cases as grade I and II respectively. The male-to-female ratio of patients with IgA nephropathy was 2:1. Recurrent gross hematuria was identified in 52 cases (61.9\%) with IgA nephropathy, with hypertension identified in 12 cases

Table 3. Clinical Status and Glomerular Morphology in Nephrotic Syndrome

\begin{tabular}{|c|c|c|c|c|c|}
\hline Glomerular morphology & No. of cases & $\begin{array}{l}\text { Microscopic } \\
\text { Hematuria }\end{array}$ & $\begin{array}{l}\text { Serum Albumin } \\
<2.5 \mathrm{gm} / \mathrm{dL}\end{array}$ & Hypertension & $\begin{array}{c}\text { Complement } 3 \text { (C3) } \\
\text { below normal }\end{array}$ \\
\hline MCD & 68 & 21 & 51 & 9 & 1 \\
\hline IgM Nephropathy & 11 & 3 & 6 & 2 & 0 \\
\hline FSGS & 9 & 6 & 4 & 3 & 1 \\
\hline MPGN & 7 & 6 & 1 & 1 & 5 \\
\hline IgA Nephropathy & 5 & 4 & 3 & 1 & 0 \\
\hline DMPGN & 4 & 2 & 0 & 1 & 0 \\
\hline Membranous glomerulopathy & 4 & 4 & 1 & 0 & 1 \\
\hline Postinfectious glomerulonephropathy & 3 & 3 & 0 & 1 & 3 \\
\hline Henoch-Schönlein purpura nephritis & 1 & 1 & 0 & 0 & 0 \\
\hline Hepatitis B Virus Associated & 1 & 1 & 1 & 0 & 1 \\
\hline Total (\%) & 102 & $48(47.1)$ & $61(59.8)$ & $16(15.7)$ & $12(11.8)$ \\
\hline
\end{tabular}

Abbreviations: MCD, Minimal change disease; IgM nephropathy, Immunoglobulin M nephropathy; FSGS, Focal segmental glomerulosclerosis; MPGN, Membranoproliferative glomerulonephritis DMPGN, Diffuse mesangial proliferative glomerulonephritis.

Table 4. Clinical Features According to Histological Grades in IgA Nephropathy

\begin{tabular}{|c|c|c|c|c|}
\hline & \multicolumn{4}{|c|}{ Histological grading* } \\
\hline & I $(n=9)$ & $\|(n=9)$ & III $(n=46)$ & IV $(n=20)$ \\
\hline Age (years) & $11.3 \pm 3.4$ & $11.3 \pm 3.4$ & $11.5 \pm 3.0$ & $10.7 \pm 3.2$ \\
\hline $\operatorname{Sex}(M: F)$ & $8: 1$ & $6: 3$ & 29:17 & $13: 7$ \\
\hline Mean duration of disease Before biopsy(months) & $5.3 \pm 6.5$ & $11.7 \pm 19.7$ & $8.7 \pm 10.8$ & $10.1 \pm 15.7$ \\
\hline Gross hematuria & 6 & 7 & 32 & 7 \\
\hline Microscopic hematuria & 7 & 6 & 41 & 18 \\
\hline Proteinuria (Urine dipstick $\geq 3+$ ) & 1 & 3 & 14 & 9 \\
\hline Hypertension & 3 & 2 & 4 & 3 \\
\hline Renal impairment (GFR<60 mL/min/1.73m²) & 0 & 0 & 3 & 0 \\
\hline \multicolumn{5}{|l|}{ Proteinuria } \\
\hline $1-2 \mathrm{gm} / 24 \mathrm{hr}$ & 0 & 1 & 9 & 2 \\
\hline$>2 \mathrm{gm} / 24 \mathrm{hr}$ & 0 & 3 & 6 & 7 \\
\hline
\end{tabular}

*Haas M: Histologic subclassification of IgA nephropathy: A Clinicopathologic study of 244 cases, Am J of Kidney disease 28:829-42, 1997.

Abbreviation: GFR, Glomerular filtration rate. 
(14.2\%), proteinuria $>1.0 \mathrm{gm} / 24 \mathrm{hr}$ in 28 cases $(33.3 \%)$, and renal impairment in 3 cases (3.6\%).

\section{Discussion}

In this study, we examined the anthropometric characteristics of patients who underwent renal biopsy along with their indications for renal biopsy and common histopathological findings. Our study revealed that the asymptomatic urinary abnormality is the most common clinical diagnosis and IgA nephropathy is the most common primary renal disease.

Renal biopsy in pediatric populations was introduced for diagnosing kidney disease in 1951 and has become a routine procedure in pediatric nephrology ${ }^{7,8)}$. Over a 27-year period, 345 children underwent renal biopsy in our hospital and we identified an overall failure rate of renal biopsy of 1.2\% (4/345), which is comparable to previously published rates ${ }^{9,10)}$. Among our study sample, the male-to-female ratio was $1.8: 1$, a rate of male predominance that was comparable to previously reported sex-specific differences in prevalence ${ }^{11)}$. Printza et al. ${ }^{12)}$ reported that $48.0 \%$ of pediatric patients who underwent renal biopsy were 11-14 years old, with 31.0 $\%$ between the ages of 6-10 years. We identified a similar age distribution, with 131 patients $(41.2 \%)$ between the ages of 11-15 years and 129 (40.5\%) between the ages of 6-10 years.

Previous studies have identified idiopathic nephrotic syndrome to the most common indication for renal biopsy ${ }^{13-16)}$. In contrast, $53.1 \%$ of patients in our study populations underwent renal biopsy to evaluate gross hematuria and/ or microscopic hematuria. Since 1998 in South Korea, more than 4 million students have participated in annual largescale urine screening programs; additionally, many schoolchildren with asymptomatic hematuria and/or proteinuria were detected and their diagnoses confirmed through renal biopsy $^{17)}$. In current study, asymptomatic urinary abnormalities were clinically diagnosed before renal biopsy in 114 patients (35.9\%), with 89 patients (27.9\%) diagnosed as having IgA nephropathy after renal biopsy.

Nephrotic syndrome can be associated with various types of glomerulonephritis and, therefore, an accurate histopathological identification of the glomerular lesion is essential to establishing a correct diagnosis and providing the appropriate treatment. Kim et al. ${ }^{18)}$ reported that among 52 pediatric patients with nephrotic syndrome, $90.4 \%$ were diagnosed with primary nephrotic syndrome and $9.6 \%$ with secondary nephrotic syndrome. In our study, primary nephrotic syndrome was identified in $95.1 \%$ of cases, with secondary nephrotic syndrome identified in $4.9 \%$ of cases. According to Choi et al. ${ }^{11)}$, minimal change disease (MCD) was identified in $72.7 \%$ of all pediatric patients. A similar prevalence of MCD (70.1\%) was found in our study. In addition, Keeping in mind the controversy regarding the nature of these IgM deposits ${ }^{19-21)}$, MCD with mesangial IgM deposit was identified in $16.2 \%$ in our study. Microscopic hematuria is present at diagnosis in $20 \%$ to $30 \%$ of children with MCD but rarely persists; macroscopic hematuria occurs in less than $1 \%$ of children with $\mathrm{MCD}^{22)}$. According to White et al. ${ }^{23)}$, microscopic hematuria was present in 13.0 $\%$ of cases with MCD. In our study, we identified hematuria in $30.8 \%$ of cases with MCD. Serum albumin levels usually fall below $2.0 \mathrm{~g} / \mathrm{dL}$ and may be less than $1.0 \mathrm{~g} / \mathrm{dL}$ in steroid sensitive nephrotic syndrome ${ }^{24)}$. Kim et al. ${ }^{18)}$ reported that the prevalence of low serum albumin levels $<2.5 \mathrm{~g} / \mathrm{dL}$ was $78.4 \%$ in children with nephrotic syndrome. In our study, we found that $59.8 \%$ of children with nephrotic syndrome and $75.0 \%$ of children with MCD showed low serum albu$\min <2.5 \mathrm{~g} / \mathrm{dL}$. Elevated systolic and diastolic blood pressure are initially present in 5-20\% of children with MCD, but hypertension usually does not persist ${ }^{22)}$. However, the frequency of hypertension occurring in other types of nephrotic syndrome is as high as $80 \%{ }^{22}$. In this study, only 15.7 $\%$ of all cases of nephrotic syndrome presented hypertension at diagnosis.

IgA nephropathy is the most prevalent form of glomerulonephritis worldwide. However, according to a large biopsy series, the prevalence of IgA nephropathy is influenced by significant race- and ethnicity-specific variation ${ }^{6)}$. In their single center study, Choi et al. ${ }^{11)}$ reported a prevalence rate of IgA nephropathy of $18.2 \%$ in children, a prevalence rate similar to that reported in Japan ${ }^{25)}$. However, the prevalence rate of IgA nephropathy in current study was $27.9 \%$, relatively high compared to other studies. The higher prevalence of IgA nephropathy in our study reflects that many school children with asymptomatic urinary abnormalities have been detected through annual mass urinary screening, which started in 1998 in South Korea ${ }^{17)}$. This screening pro- 
gram allows us to employ an active approach for evaluating children with persistent hematuria. Actually, the numbers of renal biopsy in our hospital increased annually compared to before 2000 .

A majority of children with IgA nephropathy present with gross hematuria. In other patients, microscopic hematuria and/ or proteinuria are the only signs at presentation $^{26)}$. Asymptomatic gross hematuria was identified in $61.9 \%$ of children with IgA nephropathy in our study and the prevalence of gross hematuria is similar to other studies ${ }^{27,28)}$. Although IgA nephropathy does not lead to significant renal damage, progressive renal damage develops in $20-30 \%$ of patients about 20 years after disease onset. Clinical parameters indicative of a poor prognosis are massive proteinuria (>1 gm/24 hr), renal insufficiency, and hypertension $^{29)}$. The prevalence of massive proteinuria (33.3\%) and hypertension (14.2\%) in present studied population are similar to that seen in other studies ${ }^{27,28)}$. In addition, 23 of 27 patients $(85.7 \%)$ with massive proteinuria and 7 of 12 patients (58.3\%) with hypertension were diagnosed with a grade III or IV of IgA nephropathy. Because only less than $30 \%$ patients of IgA nephropathy have been follow-up more than 5 years, we could not identify a significant relationship between the above mentioned 3 clinical parameters and prognosis in children with IgA nephropathy.

In conclusion, the most common clinical diagnosis was asymptomatic urinary abnormalities, followed by nephrotic syndrome and acute glomerulonephritis. Primary glomerular disease is the most common histological finding, with IgA nephropathy being the most common histopathological lesion, followed by MCD. Our study demonstrates the importance of percutaneous renal biopsy in the diagnosis and treatment of pediatric renal diseases.

\section{Conflicts of interest}

No potential conflict of interest relevant to this article was reported.

\section{References}

1. Tondel C, Vikse BE, Bostad L, Svarstad E. Safety and complications of percutaneous kidney biopsies in 715 children and 8573 adults in Norway 1988-2010. Clin J Am Soc Nephrol 2012;7:1591-7.

2. Gault MH, Muehrcke RC. Renal biopsy: Current views and controversies. Nephron 1983;34:1-34.

3. Edelmann CM Jr, Greifer I. A modified technique for percutaneous needle biopsy of the kidney. J Pediatr 1967;70:81-6.

4. al Rasheed SA, al Mugeiren MM, Abdurrahman MB, Elidrissy AT. The outcome of percutaneous renal biopsy in children: An analysis of 120 consecutive cases. Pediatr Nephrol 1990;4:600-3.

5. Fuiano G, Mazza G, Comi N. Current indication for renal biopsy: A questionnaire based survey. Am J Kidney Dis 2000;35:448-57.

6. Haas M. Histologic subclassification of IgA nephropathy: A clinicopathologic study of 244 cases. Am J Kidney Dis 1997;28:829-42

7. Cameron JS. The introduction of renal biopsy into nephrology from 1901 to 1961; A paradigm of the forming of nephrology by technology. Am J Nephrol 1997;17:348-58.

8. Habib R. A story of glomerulopathies: A pathologist's experience. Pediatr Nephrol 1993;7:336-46.

9. Feneberg R, Schaefer F, Zieger B, Waldherr R, Mehls O, Schärer K. Percutaneous renal biopsy in children: a 27-year experience. Nephron 1998;79:438-46.

10. Kamitsuji H, Yoshioka K, Ito H. Percutaneous renal biopsy in children: survey of pediatric nephrologists in Japan. Pediatr Nephrol 1999;13:693-6.

11. Choi IJ, Jeong HJ, Han DS, Lee JS, Choi KH, Kang SW, et al. An analysis of 4514 cases of renal biopsy in Korea. Yonsei Med J 2001;2:247-54

12. Printza N, Bosdou J, Pantzaki A, Badouraki M, Kollios K, Ghogha Ch, et al. Percutaneous ultrasound-guided renal biopsy in children: a single center experience. Hippokratia 2011;3:258-61.

13. Lanewala A, Mubarak M, Akhter F, Aziz S, Bhatti S, Kazi Jl. Pattern of pediatric renal disease observed in native renal biopsies in Pakistan. J Nephrol 2009;22:739-46.

14. Moorani KN, Sherali AR. Histopathological pattern in childhood glomerulonephritis. J Pak Med Assoc 2010;60:1006-9.

15. Ali A, Ali MU, Akhtar SZ. Histological pattern of paediatric renal diseases in northern Pakistan. J Pak Med Assoc 2011;61:653-8.

16. Absar A, Diamond M, Sonia Y, Arshalooz R, Safia A, Waqar K, et al. Ten year experience of pediatric kidney biopsies from a single center in Pakistan. Indian J Nephrol 2010;20:190-2.

17. Park YH. School urine screening program in Korea. J Korean Soc Pediatric Nephrol 2014;18:57-63

18. Kim BK, Jeong CK, Lee JS, Yoon DJ, Choi IJ. Clinicopathological study on renal biopsies in children. J Korean Med Assoc 1981;24: 425-32.

19. Border WA. Distinguishing minimal-change disease from mesangial disorders. Kidney Int 1988;34(3):419

20. Myllymaki J, Saha H, Mustonen J, Helin H, Pasternack A. IgM nephropathy: clinical picture and long-term prognosis. Am J Kidney Dis 2003:41:343-50. 
21. Al-Eisa A, Carter JE, Lirenman DS, Magil AB. Children IgM nephropathy: comparison with minimal change disease. Nephron 1996; 72:37-43.

22. A report of the International Study of Kidney Disease in Children. Nephrotic syndrome in children: prediction of histopathology from clinical and laboratory characteristics at time of diagnosis. Kidney Int 1978;13:159.

23. White RHR. Glasgow EF. Mills RJ. Clinicopathological study of nephrotic syndrome in childhood. Lancet 1970;1:1353-9.

24. Habib R, Kleinknecht C. The primary nephrotic syndrome of childhood. Classification and clinicopathologic study of 406 cases. Pathology annual 1971;6:417-74.

25. Kitajima T, Murakami M, Sakai O. Clinicopathological features in the Japanese patients with IgA nephropathy. Jpn J Med 1983;22: 219-22.
26. D'Amico G, Minetti L, Ponticelli C, Fellin G, Ferrario F, Barbiano di Belgioioso $\mathrm{G}$ et al. Prognostic indicators in idiopathic IgA mesangical nephropathy. Quarterly J Med 1986;59:363-78.

27. Topaloglu R, Orhan D, Bilginer Y, Karabulut E, Ozaltin F, Duzova A, et al. Clinicopathological and immunohistological features in childhood IgA nephropathy: a single-centre experience. Clin Kidney J 2013;6:169-75.

28. Haas M, Rahman MH, Cohn RA, Fathallah-Shaykh S, Ansari A, Bartosh SM; IgA nephropathy in children and adults comparison of histologic features and clinical outcome. Nephrol Dial Transplant 2008;23:2537-45

29. Fofi C, Pecci G, Galliani M, Comunian MC, Muda AO, Pierucci A, et al. IgA nephropathy: multivariate statistical analysis aimed at predicting outcome. J Nephrol 2001;14:280-5. 